\title{
TU/e EnNHONEN

\section{A mode-matching method for three-dimensional waveguides with PMLs combined with energy conservation}

\section{Citation for published version (APA):}

Lu, X., Cao, Z., van Beurden, M., Jiao, Y., Wu, Q., \& Koonen, T. (2018). A mode-matching method for threedimensional waveguides with PMLs combined with energy conservation. Journal of Lightwave Technology, 36(23), 5573-5579. [8458412]. https://doi.org/10.1109/JLT.2018.2869701

\section{Document license:}

TAVERNE

DOI:

10.1109/JLT.2018.2869701

Document status and date:

Published: 01/12/2018

\section{Document Version:}

Publisher's PDF, also known as Version of Record (includes final page, issue and volume numbers)

\section{Please check the document version of this publication:}

- A submitted manuscript is the version of the article upon submission and before peer-review. There can be important differences between the submitted version and the official published version of record. People interested in the research are advised to contact the author for the final version of the publication, or visit the $\mathrm{DOI}$ to the publisher's website.

- The final author version and the galley proof are versions of the publication after peer review.

- The final published version features the final layout of the paper including the volume, issue and page numbers.

Link to publication

\section{General rights}

Copyright and moral rights for the publications made accessible in the public portal are retained by the authors and/or other copyright owners and it is a condition of accessing publications that users recognise and abide by the legal requirements associated with these rights.

- Users may download and print one copy of any publication from the public portal for the purpose of private study or research.

- You may not further distribute the material or use it for any profit-making activity or commercial gain

- You may freely distribute the URL identifying the publication in the public portal.

If the publication is distributed under the terms of Article 25fa of the Dutch Copyright Act, indicated by the "Taverne" license above, please follow below link for the End User Agreement:

www.tue.nl/taverne

Take down policy

If you believe that this document breaches copyright please contact us at:

openaccess@tue.nl

providing details and we will investigate your claim. 


\title{
A Mode-Matching Method for Three-Dimensional Waveguides With PMLs Combined With Energy Conservation
}

\author{
Xinyang Lu ${ }^{\circledR}$, Zizheng Cao ${ }^{\circledR}$, Member, IEEE, M. C. van Beurden ${ }^{(}$, Yuqing Jiao ${ }^{\circledR}$, Member, IEEE, \\ Qingbiao Wu, and Ton Koonen ${ }^{\circledR}$, Fellow, IEEE
}

\begin{abstract}
We present an improved calculation for reflection and transmission coefficients in the context of a mode-matching method with optimization, which makes the results more reliable and accurate as the number of modes employed in the mode-matching method is increased. This method is successfully demonstrated in a series of waveguide structures.
\end{abstract}

Index Terms-Optical waveguide, mode matching, optimization, Newton method.

\section{INTRODUCTION}

W ITH high efficiency and precision, the mode-matching method (MMM, also called eigenmode expansion method) [1] is a powerful approach to deal with optical waveguide discontinuities between two $z$-invariant structures ( $z$ is the propagation direction) that are common in propagation problems [2]-[4]. It confines the boundary-value problem to the boundary conditions only by utilizing the continuity of the tangential components of the electric and magnetic fields at the interface between two $z$-invariant waveguides.

There is abundant literature on the propagation of waves in optical waveguides. In the early development of MMM, multilayer waveguides with simple structures and accurate analytic solutions were the main research subjects [5], [6] and it showed good efficiency and convergence in such applications. For example, the multi-mode interference (MMI) coupler itself is a single

Manuscript received March 8, 2018; revised June 4, 2018 and August 1, 2018; accepted September 7, 2018. Date of publication September 10, 2018; date of current version November 2, 2018. This work was supported in part by the ERC FP-7 advanced grant BROWSE (Agreement: 291632), in part by the NWO Zwaartekracht program on Integrated nanophotonics, the National Natural Science Foundation of China under Grants 61575186, 61635001, 11771393, and 11632015 , in part by the Zhejiang Natural Science Foundation under Grant LZ14A010002, and in part by the open fund from State Key Laboratory of Advanced Optical Communication Systems Networks, China. The work of X. Lu was supported by the China Scholarship Council (201606320117). (Corresponding author: M.C. van Beurden.)

$\mathrm{X}$. Lu and Q. Wu are with the School of Mathematical Sciences, Zhejiang University, Hangzhou 310027, Zhejiang, China (e-mail: luxinyang @ zju.edu.cn; qbwu@zju.edu.cn).

Z. Cao, Y. Jiao, and Ton Koonen are with the Institute for Photonic Integration, Eindhoven University of Technology, 5600 MB Eindhoven, The Netherlands (e-mail: Z.Cao@tuel.nl; Y.Jiao@tue.nl; A.M.J.Koonen@tue.nl).

M. C. van Beurden is with the Department of Electrical Engineering, Eindhoven University of Technology, 5600 MB Eindhoven, The Netherlands (e-mail: M.C.v.Beurden@tue.nl).

Color versions of one or more of the figures in this paper are available online at http://ieeexplore.ieee.org.

Digital Object Identifier 10.1109/JLT.2018.2869701 segment, which is a good engineering example of MMM used in silicon photonics [7]. Subsequently, this method was extended to deal with three-dimensional waveguides by slicing the structure in a number of segments in which the permittivity profile remains $z$-invariant. In each segment, the field is expanded in a set of modes, which are calculated by a mode solver [8], [9]. By applying the tangential continuity conditions and the mode orthogonality relations [10], the different segments can be related by a scattering matrix [11]. The high computational efficiency of MMM is attributed to the use of the mode expansion instead of a spatial discretization [12].

In practical applications, much more attention is paid to the simulation of open waveguide structures in which the solution domain is infinite, which is mathematically challenging in numerical calculations. Currently, perfectly matching layers (PML) terminated by a perfectly reflecting boundary (PRB) condition are widely used [11]. Under the PML condition, the continuous spectrum of non-guided modes can be approximated by a series of discrete complex modes. Although the modal orthogonality and normalization of these modes are not as critical as guided modes, all modes can be dealt with, by similar analytical and numerical techniques [13].

The combination of MMM and a mode solver using PMLs and PRBs are well suited to transmission problems [7], but there are challenges when dealing with reflection and scattering. In the actual calculation, we get unphysical reflection and transmission coefficients that seriously violate the conservation of energy in some cases (as will be shown in Fig. 6 later on). In the basic concept of MMM, an infinite number of complex modes are employed in the calculation to satisfy the tangential continuity, while in practice the number of modes is finite. Consequently, the continuity condition fails and there is a field mismatch error [9]. However, this does not mean that the mismatch error always reduces for an increasing number of modes, due to the unphysical nature of the modes calculated by mode solvers that exploit PMLs. Those unphysical modes, which increase exponentially in the PML cladding, increase the condition number of the coefficient matrix and make the results unreliable. To make the algorithm more stable, we introduce an $L_{2}$-norm constraint based on the conservation of energy and transform the linear equations into an optimization problem. In spite of the increasing computational overhead, the results of the improved method are much more robust with a relatively 


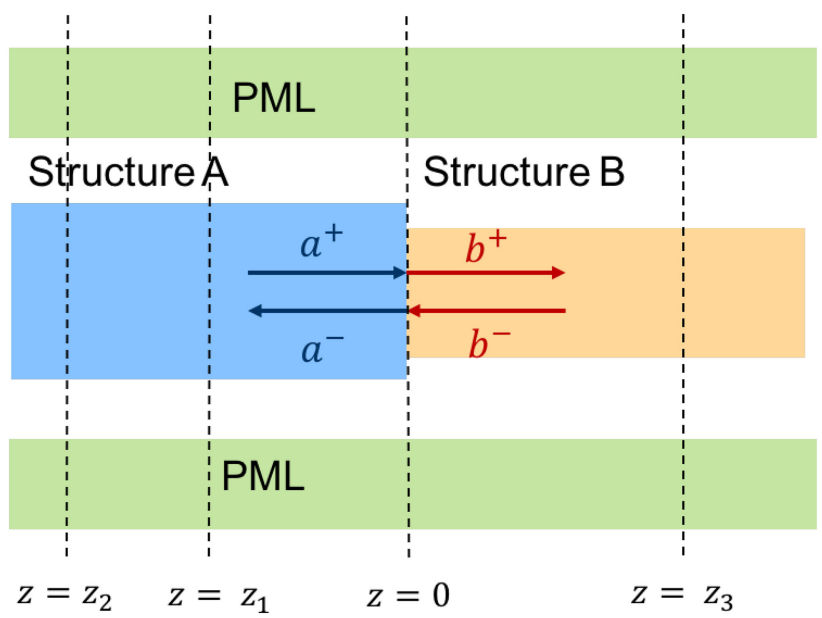

Fig. 1. The waveguide consist of structure A and B, which are both $z$-invariant and surrounded by PML. They The propagation direction is $z$ and there is a permittivity discontinuity interface at $z=0$.

small field-mismatch error and do not vary drastically with the number of modes and relative index contrast.

\section{General Formularization OF THE MODE-MATCHING MeTHOD}

Assume the propagation direction is along the $z$ direction and the transverse cross section is the $x y$ plane. The modematching interface is characterized by two distinct waveguide sections (Fig. 1) with $z$-invariant permittivity profiles and both are surrounded by PML in the transverse plane. The absorption properties of the PML regions are controlled by three parameters, which definition can be found in [14]. Assuming that $N$ modes are employed on both sides, the transverse fields can be written as a linear superposition of the forward (denoted by '+') and the backward (denoted by '-') modes with in general complex propagation coefficients $\beta_{k}$ as:

$$
\begin{aligned}
\boldsymbol{E}_{t} & =\sum_{k=1}^{N}\left(a_{k}^{+} e^{-j \beta_{k} z}+a_{k}^{-} e^{j \beta_{k} z}\right) \boldsymbol{e}_{t, k}(x, y), \\
\boldsymbol{H}_{t} & =\sum_{k=1}^{N}\left(a_{k}^{+} e^{-j \beta_{k} z}-a_{k}^{-} e^{j \beta_{k} z}\right) \boldsymbol{h}_{t, k}(x, y),
\end{aligned}
$$

where the subscript $t$ denotes the transverse component. $\boldsymbol{e}_{t}$ and $\boldsymbol{h}_{t}$ are the transverse electric and magnetic fields and $a_{k}^{ \pm}$are the coefficients of the forward and backward traveling modes.

The continuity of the tangential electric and magnetic fields at the discontinuous interface $z=0$ are expressed as follows [9], [11]:

$$
\begin{aligned}
& \sum_{k=1}^{N}\left(a_{k}^{+}+a_{k}^{-}\right) \boldsymbol{e}_{t, k}^{A}(x, y)=\sum_{k=1}^{N}\left(b_{k}^{+}+b_{k}^{-}\right) \boldsymbol{e}_{t, k}^{B}(x, y), \\
& \sum_{k=1}^{N}\left(a_{k}^{+}-a_{k}^{-}\right) \boldsymbol{h}_{t, k}^{A}(x, y)=\sum_{k=1}^{N}\left(b_{k}^{+}-b_{k}^{-}\right) \boldsymbol{h}_{t, k}^{B}(x, y),
\end{aligned}
$$

where the superscripts $A$ and $B$ denote the waveguide sections for $z<0$ and $z>0$, respectively.
In the mode solver applied here, the PML boundary condition is implemented via stretching coordinates. The introduction of the imaginary part in the coordinates reduces the field reciprocity relation defined for the exact solution of Maxwell's equations to a sesquilinear form instead of a true inner product since this form does not satisfy conjugate symmetry and positivedefiniteness. The theoretical orthogonality derived from the field reciprocity relation [10], is not a stable relation in the numerical approximation and therefore we do not exploit the orthogonality. Similar to the solution of the above equations in [11] and [15], we can integrate the $z$ component of Eq. $(1 \mathrm{a}) \times\left(\boldsymbol{h}_{t k}^{A}+\boldsymbol{h}_{t k}^{B}\right)$ and $\left(\boldsymbol{e}_{t k}^{A}+\boldsymbol{e}_{t k}^{B}\right) \times$ Eq. (1b) over the interface at $z=0$, respectively, and obtain

$$
\left[\begin{array}{cc}
\boldsymbol{D}^{1} & \boldsymbol{D}^{1} \\
\boldsymbol{D}^{3} & -\boldsymbol{D}^{3}
\end{array}\right]\left[\begin{array}{l}
\boldsymbol{a}^{+} \\
\boldsymbol{a}^{-}
\end{array}\right]=\left[\begin{array}{cc}
\boldsymbol{D}^{2} & \boldsymbol{D}^{2} \\
\boldsymbol{D}^{4} & -\boldsymbol{D}^{4}
\end{array}\right]\left[\begin{array}{l}
\boldsymbol{b}^{+} \\
\boldsymbol{b}^{-}
\end{array}\right]
$$

where the block matrices $\boldsymbol{D}^{i} \in \mathbb{C}^{N \times N}(i=1,2,3,4)$ are determined by the inner product of the field vectors as follows:

$$
\begin{aligned}
\boldsymbol{D}^{1}(j, i) & =\left\langle\boldsymbol{e}_{i}^{A}, \boldsymbol{h}_{j}^{A}+\boldsymbol{h}_{j}^{B}\right\rangle \\
\boldsymbol{D}^{2}(j, i) & =\left\langle\boldsymbol{e}_{i}^{B}, \boldsymbol{h}_{j}^{A}+\boldsymbol{h}_{j}^{B}\right\rangle, \\
\boldsymbol{D}^{3}(i, j) & =\left\langle\boldsymbol{e}_{i}^{A}+\boldsymbol{e}_{i}^{B}, \boldsymbol{h}_{j}^{A}\right\rangle \\
\boldsymbol{D}^{4}(i, j) & =\left\langle\boldsymbol{e}_{i}^{A}+\boldsymbol{e}_{i}^{B}, \boldsymbol{h}_{j}^{A}\right\rangle, \\
\left\langle\boldsymbol{e}_{i}, \boldsymbol{h}_{j}\right\rangle & =\frac{1}{2} \iint\left(\boldsymbol{e}_{i} \times \boldsymbol{h}_{j}\right) \cdot \boldsymbol{z} d s .
\end{aligned}
$$

We can rewrite Eq. (2) as

$$
\begin{aligned}
{\left[\begin{array}{l}
\boldsymbol{b}^{+} \\
\boldsymbol{b}^{-}
\end{array}\right] } & =\frac{1}{2}\left[\begin{array}{cc}
\left(\boldsymbol{D}^{1}\right)^{-1} & \left(\boldsymbol{D}^{4}\right)^{-1} \\
\left(\boldsymbol{D}^{2}\right)^{-1} & -\left(\boldsymbol{D}^{4}\right)^{-1}
\end{array}\right]\left[\begin{array}{cc}
\boldsymbol{D}^{1} & \boldsymbol{D}^{1} \\
\boldsymbol{D}^{3} & -\boldsymbol{D}^{3}
\end{array}\right]\left[\begin{array}{l}
\boldsymbol{a}^{+} \\
\boldsymbol{a}^{-}
\end{array}\right] \\
& \triangleq \boldsymbol{D}\left[\begin{array}{l}
\boldsymbol{a}^{+} \\
\boldsymbol{a}^{-}
\end{array}\right]
\end{aligned}
$$

where $\boldsymbol{D}$ denotes the transfer matrix across the discontinuous interface.

\section{COMBINATION OF MMM AND OPTIMIZATION}

Eq. (5) can be rewritten as follows:

$$
\left[\begin{array}{cc}
\boldsymbol{I} & -\boldsymbol{D}_{12} \\
\mathbf{0} & \boldsymbol{D}_{22}
\end{array}\right]\left[\begin{array}{c}
\boldsymbol{b}^{+} \\
\boldsymbol{a}^{-}
\end{array}\right]=\left[\begin{array}{cc}
\boldsymbol{D}_{11} & \mathbf{0} \\
-\boldsymbol{D}_{21} & \boldsymbol{I}
\end{array}\right]\left[\begin{array}{l}
\boldsymbol{a}^{+} \\
\boldsymbol{b}^{-}
\end{array}\right]
$$

where $\boldsymbol{I} \in \mathbb{R}^{N \times N}$ is the identity matrix and $\boldsymbol{D}=\left[\begin{array}{ll}\boldsymbol{D}_{11} & \boldsymbol{D}_{12} \\ \boldsymbol{D}_{21} & \boldsymbol{D}_{22}\end{array}\right]$.

For the two $z$-invariant semi-infinite waveguides, we assume the forward fundamental mode is launched, so the coefficients of the input field are given by the vector $\boldsymbol{a}^{+}=[1,0, \ldots, 0]^{T}$ and there is only this source in the structure $A$, so there is no backward wave in structure $B$, i.e. $\boldsymbol{b}^{-}=\mathbf{0}$. Then Eq. (6) can be expressed in a more compact way as

$$
M \cdot x=f,
$$

where $\boldsymbol{M}=\left[\begin{array}{cc}\boldsymbol{I} & -\boldsymbol{D}_{12} \\ \mathbf{0} & \boldsymbol{D}_{22}\end{array}\right]$ and $\boldsymbol{f}=\left[\begin{array}{cc}\boldsymbol{D}_{11} & \mathbf{0} \\ -\boldsymbol{D}_{21} & \boldsymbol{I}\end{array}\right]\left[\begin{array}{c}\boldsymbol{a}^{+} \\ \boldsymbol{b}^{-}\end{array}\right]$are known, while $\boldsymbol{x}=\left[\begin{array}{c}\boldsymbol{b}^{+} \\ \boldsymbol{a}^{-}\end{array}\right]$is unknown.

We realize that Eq. (5) is not strictly an equality in practical conditions and according to the conservation of energy at the interface at $z=0$ (where every mode has been normalized and 
its power equals 1 ) if there are infinitely many modes employed, we have

$$
1=\left\|\boldsymbol{a}^{+}\right\|^{2}+\left\|\boldsymbol{b}^{-}\right\|^{2}=\left\|\boldsymbol{a}^{-}\right\|^{2}+\left\|\boldsymbol{b}^{+}\right\|^{2},
$$

where $\|\cdot\|$ denotes the Euclidean norm on a vector space.

Here, we present the introduction of the $L_{2}$-norm constraint based on Eq. (8) and solve Eq. (7) as a constrained optimization problem:

$$
\min _{\|\boldsymbol{x}\|=g} F(\boldsymbol{x})=\|\boldsymbol{M} \cdot \boldsymbol{x}-\boldsymbol{f}\|^{2}
$$

Next, we simplify the above problem based on the concept of penalty-function methods [16] as follows:

$$
\min _{\boldsymbol{x}} F(\boldsymbol{x})=\|\boldsymbol{M} \cdot \boldsymbol{x}-\boldsymbol{f}\|^{2}+c(\|\boldsymbol{x}\|-g)^{2}
$$

where $c$ is the penalty parameter. Here, we set $c=1000$.

Then we use Newton's method to solve the above problem. At first, we transfer the objective function $F(\boldsymbol{x})$ in the complex field to the equivalent function $F^{r}(\boldsymbol{y})$ in the real field:

$$
F^{r}(\boldsymbol{y})=\left\|\boldsymbol{M}^{r} \boldsymbol{y}-\boldsymbol{f}^{r}\right\|^{2}+c(\|\boldsymbol{y}\|-g)^{2},
$$

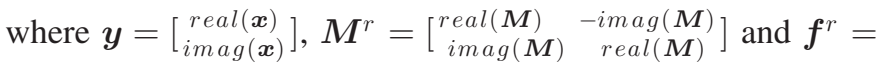
$\left[\begin{array}{c}\operatorname{real}(\boldsymbol{f}) \\ \operatorname{imag}(\boldsymbol{f})\end{array}\right]$.

It is easy to get the Hessian matrix of $F^{r}$ as follows:

$$
\nabla^{2} F^{r}(\boldsymbol{y})=2\left(\boldsymbol{M}^{r}\right)^{T} \cdot \boldsymbol{M}^{r}+2 c\left(1-\frac{g}{\|\boldsymbol{y}\|}\right) \boldsymbol{I}+2 c \frac{\boldsymbol{y} \boldsymbol{y}^{T}}{\|\boldsymbol{y}\|^{3}} .
$$

For any vector $\overline{\boldsymbol{y}} \in \mathbb{R}^{4 N}$ and vector $\boldsymbol{y} \in \mathbb{R}^{4 N}$, which satisfies $\|\boldsymbol{y}\| \approx g$,

$$
\overline{\boldsymbol{y}}^{T} \nabla^{2} F^{r}(\boldsymbol{y}) \overline{\boldsymbol{y}} \approx 2\left(\boldsymbol{M}^{r} \overline{\boldsymbol{y}}\right)^{T}\left(\boldsymbol{M}^{r} \overline{\boldsymbol{y}}\right)+2 c \frac{\left(\boldsymbol{y}^{T} \overline{\boldsymbol{y}}\right)^{T}\left(\boldsymbol{y}^{T} \overline{\boldsymbol{y}}\right)}{\|\boldsymbol{y}\|^{3}} \geq 0
$$

The above inequality shows that $F^{r}(\boldsymbol{y})$ is a locally convex function. According to the optimization theory in [16], we can get the minimum of $F^{r}$ in the local region where $\|\boldsymbol{y}\| \approx g$.

To evaluate the performance of the improved MMM, the field mismatch error $\Delta(F)$ is defined as follows:

$$
\Delta(F)=\frac{\max \left(\left|\boldsymbol{F}^{\text {input }}+\boldsymbol{F}^{\text {refl }}-\boldsymbol{F}^{\text {tran }}\right|^{2}\right)}{\max \left(\left|\boldsymbol{F}^{\text {input }}\right|^{2}\right)}
$$

Here $\boldsymbol{F}$ can be the electric or magnetic field. $\boldsymbol{F}^{\text {input }}$ and $\boldsymbol{F}^{\text {refl }}$ denote the input excitation field and the reflected field in structure A, respectively, while $\boldsymbol{F}^{\text {tran }}$ denotes the transmitted field in structure B. In the next section we will compare the errors obtained from solving Eq. (7) by directly solving the equations for the general MMM and from Eq. (11) for MMM-O.

\section{VALidation of the Proposed Algorithm}

For Eq. (7), there is a simplified calculation (MMM-S) at the expense of accuracy. In the simplified algorithm, the reflected field is assumed to have the same profile as the incoming field. In this section, we present the mismatch errors calculated by MMM-O and compare them with the MMM (In MMM, Eq. (7)

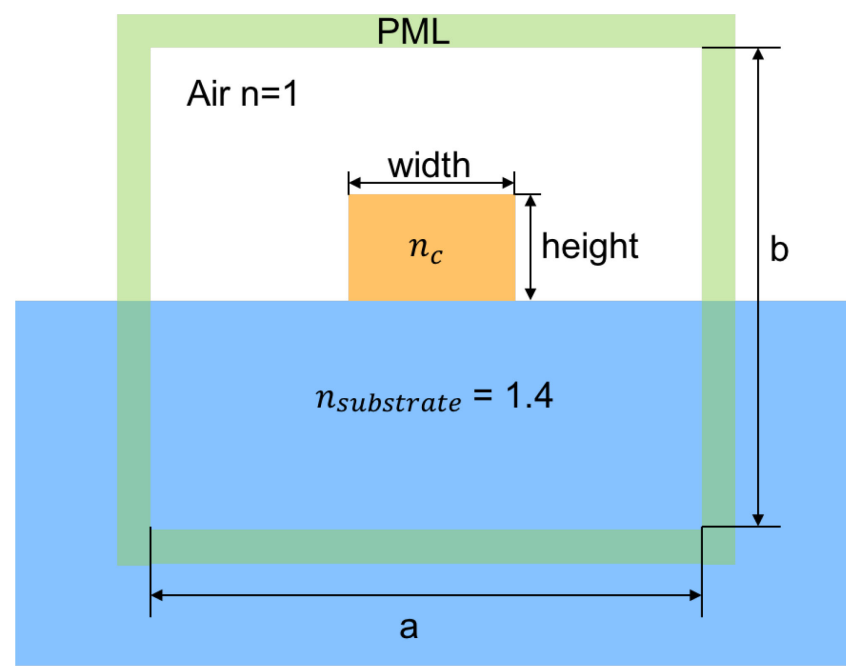

Fig. 2. The three waveguide structures have similar profile of permittivity with different width.

is solved directly by matrix inversion) and MMM-S. Here we define three waveguide structures with different width of the core illustrated in Fig. 2:

- Structure I: height $=0.3 \mu \mathrm{m}$, width $=0.4 \mu \mathrm{m}$, $n_{c}=3.18$

- Structure II: height $=0.3 \mu \mathrm{m}$, width $=0.6 \mu \mathrm{m}$, $n_{c}=3.18$

- Structure III: height $=0.3 \mu \mathrm{m}$, width $=1.2 \mu \mathrm{m}$, $n_{c}=3.18$

- Structure IV: height $=0.3 \mu \mathrm{m}$, width $=1.2 \mu \mathrm{m}$, $n_{c}=5.18$

The computational region without PML is $4 \mu \mathrm{m} \times 4 \mu \mathrm{m}$ and the PML parameters are set as explained in [17] and we let $\alpha_{j, \max }=2, j=1,2,3,4$. They control the absorption properties of the PML regions. The modes of every $z$-invariant structure are calculated by a vector finite element mode solver with rectangular elements. The algorithm and formulas of this mode solver are based on Chapter 8 in [18].

\section{A. Small Transverse Discontinuity}

First, we chose Structure I as structure A and Structure II as structure B. The width discontinuity between them is relatively small. The fundamental mode of structure A is injected at $z=z_{1}$. We calculate the reflection coefficients at $z=z_{2}$ and transmission coefficients at $z=z_{3}$. In all the examples below, we set $z_{1}=-5 \mu \mathrm{m}, z_{2}=-10 \mu \mathrm{m}$ and $z_{3}=10 \mu \mathrm{m}$. Fig. 3 shows the sum of squares of transmission and reflection coefficients of these three methods at the discontinuous interface. The figure indicates that the results of MMM-O and MMM-S satisfy the energy conservation well, while there are serious deviations in MMM. Fig. 4 demonstrates that MMM-O has a smaller error and better convergence than MMM-S. 




Fig. 3. The variation of the total energy of reflection and transmission with the number of employed modes.

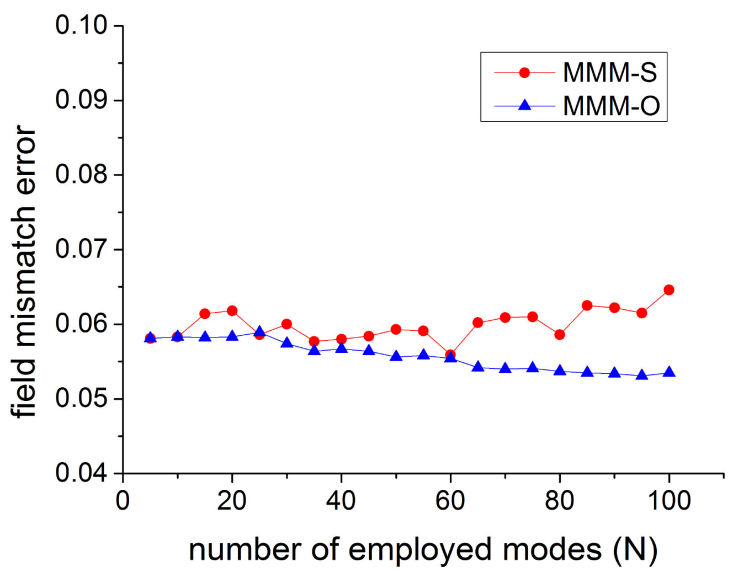

Fig. 4. The variation of the field mismatch error with the number of employed modes.

\section{B. Large Transverse Discontinuity}

Then, we replace Structure II with III, which aggravates the discontinuity at the interface $z=0$. The difference in the profiles of permittivity leads to a different distribution of modes, which has great influence on the coefficient matrix $M$ in Eq. (7).

Fig. 5 shows the mode index growth trend in the condition number of $\boldsymbol{M}$ (defined as $\left\|\boldsymbol{M}^{-1}\right\| \cdot\|\boldsymbol{M}\|$ ) along with the number of employed modes $(N)$. The high condition number implies the unreliability of matrix inversion [19], which is reflected by the large deviations in MMM. The high-index contrast can also cause a significantly large error. So the $\Delta(F)$ is larger in total than that in the small refractive-index contrast case. In the small transverse discontinuity case, the penalty function in MMM-O and the reduced dimension in MMM-S are effective to avoid the ill-conditioning of the coefficient matrix. However, only MMM-O exhibits good performance in the large transverse discontinuity case. Fig. 6 shows that good stability of MMM-O is obtained as the number of employed modes increases, while the overall error of MMM and MMM-S becomes very large and has a diverging trend. As the number of modes increases, MMM-O exhibits a better convergence. From the perspective

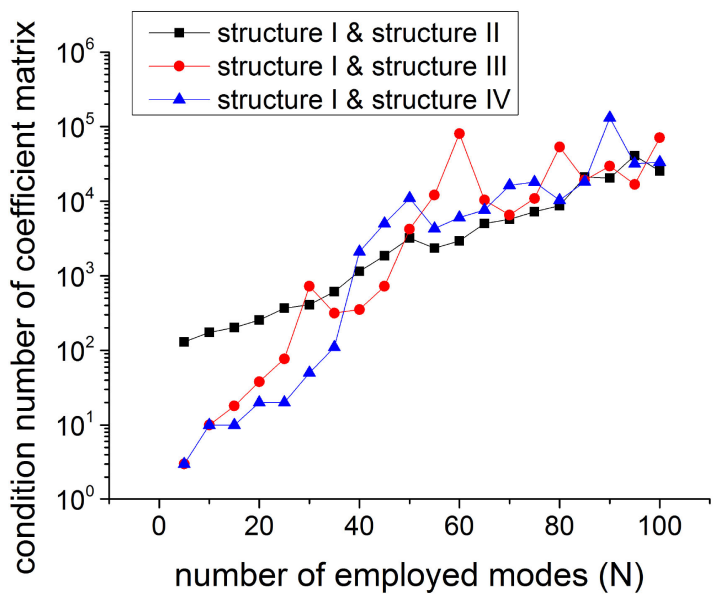

Fig. 5. The variation of the condition number of coefficient matrix $M$ versus the number of employed modes.

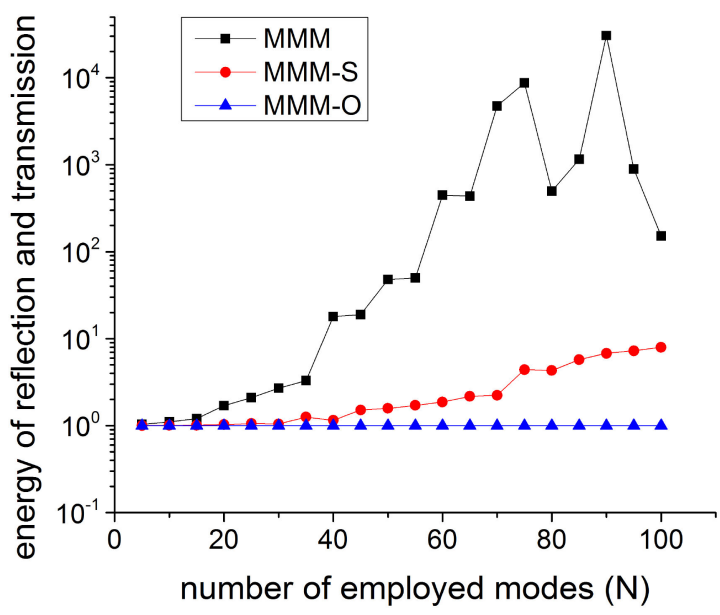

Fig. 6. The variation of the total energy of reflection and transmission versus the number of employed modes.

of error and stability, MMM-O is clearly better than the other two methods. However, MMM-O has a serious shortcoming, its computational cost. To reduce the computational complexity, we replace Newton's method with a quasi-Newton BFGS algorithm with Armijo line search. Nevertheless, its computational effort is still several times higher than that of the other two methods. This conclusion can be illustrated by Fig. 7. It demonstrates the CPU times of the example in Section IV-B calculated by the three methods respectively.

A PML is an artificial material and modes that exist within the PML are nonphysical. The PML region is lossy, therefore the orthogonality of nonphysical modes does not always hold, which causes the noise and singularity of the coefficients matrix. There may be some nonphysical modes used to calculate the scattering parameters if the number of modes we choose is more than the number of guided modes. So it does not mean that the field mismatch error reduces with the increasing number of modes, as shown in Fig. 4. In cases for which we do not know the most suitable number of employed modes, the stability of the algorithm with $N$ is especially necessary and important. 


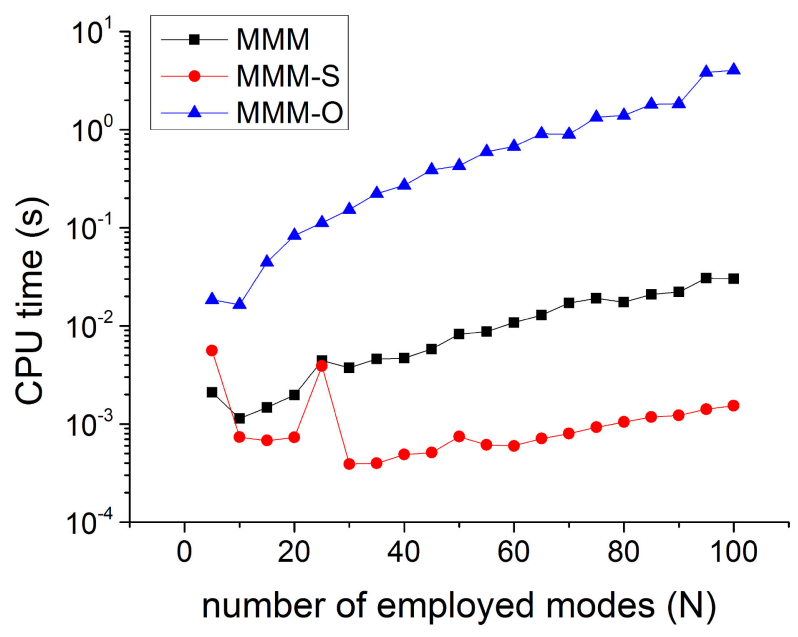

Fig. 7. The variation of the CPU time versus the number of employed modes when calculating the example in Section IV-B.



Fig. 8. The variation of the normalized energy of transmission and reflection with the number of employed modes where Structure A is Structure I and Structure B is Structure III.

\section{Comparison With FDTD}

We provide two examples to show the accuracy of MMMO through the comparison with FDTD (Lumerical FDTD Solution [20]). We choose stabilized PML parameters in FDTD for more reliable results. However, the FDTD results are considerably mesh dependent, and therefore not suitable as a true benchmark, mainly because the optimal mesh size is not known. Instead, the FDTD results should be seen as an estimation for the order of magnitude of the reflection and transmission coefficients. The first case is the large transverse discontinuity case in Section IV-B (Fig. 8 shows the comparison between MMM-O and FDTD for Structure I and Structure III). As to the second case, we keep Structure I as structure A and choose Structure IV as structure B (Fig. 14 compared FDTD shows the comparison between MMM-O and FDTD for Structure I and Structure IV). The two figures show that the results of MMM-O converge to that of FDTD well. Compared with FDTD, especially when computing long-distance transmission, the efficiency of MMM$\mathrm{O}$ is still considerable.



Fig. 9. The variation of the total energy of reflection and transimision with the number of employed modes where Structure A is Structure I and Structure B is Structure IV.

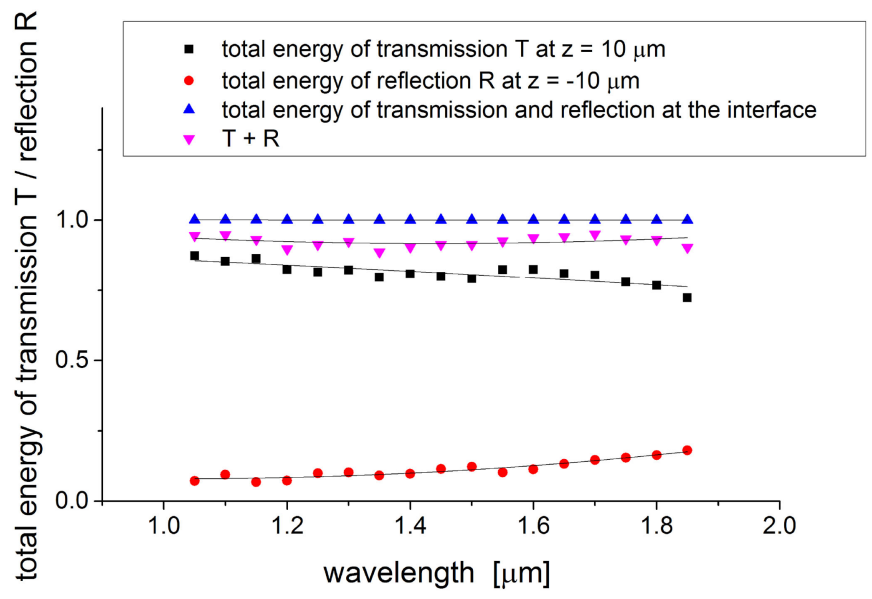

Fig. 10. The variation of the normalized energy of transmission and reflection as a function of the wavelength $\lambda$.

\section{Transmission and Reflection as a Function of the Wavelength}

This time we choose Structure IV as structure A and Structure I as structure B. We change the wavelength $\lambda$ from $1.05 \mu \mathrm{m}$ to $1.85 \mu \mathrm{m}$ with step $\Delta \lambda=0.5 \mu \mathrm{m}$. The energy of transmission and reflection of the wave propagating in the structure are shown in Fig. 10. According to the trend line, we can conclude that the transmission decreases and reflection increases with the increase in wavelength. The total energy of transmission and reflection at the discontinuous interface reflects the validation of energy conservation in MMM-O. The energy at $z=10 \mu \mathrm{m}$ and $z=-10 \mu \mathrm{m}$ shows that the loss of light in the PML, which corresponds to the radiation loss for the open waveguide, is stable as the wavelength increases except for some fluctuations.

\section{E. The Coupling Between Fiber and SOI}

To prove the practicality of the algorithm, we consider a more realistic case, in which the input waveguide (structure A) is a conventional single-mode optical fiber coupled to a silicon-oninsulator waveguide (SOI, structure B). The design of SOI is 


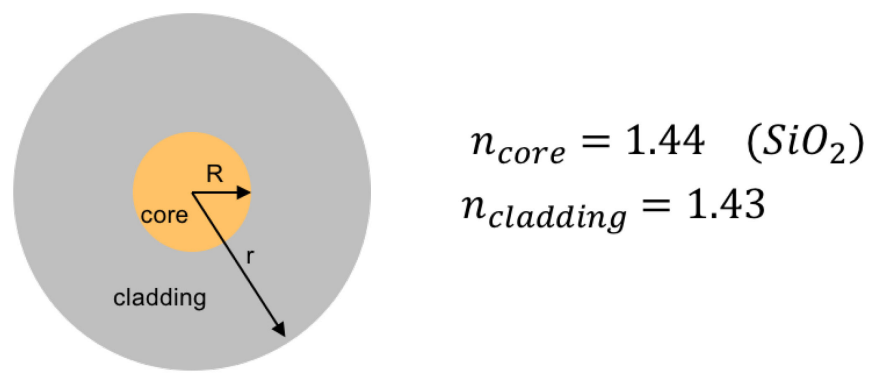

Fig. 11. The size and refractive index of the single mode optical fiber as structure A.

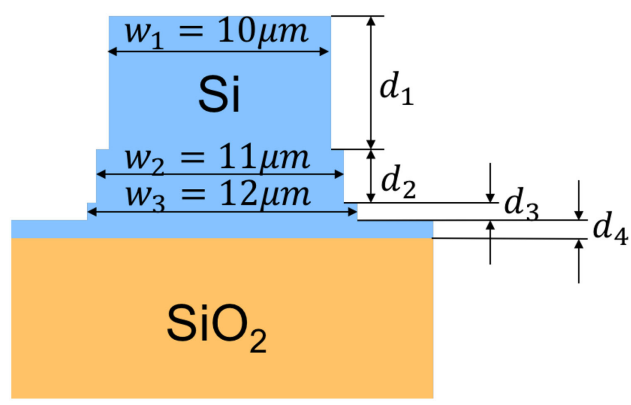

$$
\begin{aligned}
& n_{S i}=1.44 \\
& n_{S i O_{2}}=3.48 \\
& d_{1}=6 \mu m \\
& d_{2}=2.5 \mu m \\
& d_{3}=0.7 \mu m \\
& d_{4}=0.8 \mu m
\end{aligned}
$$

Fig. 12. The size and refractive index of the SOI, as structure B.

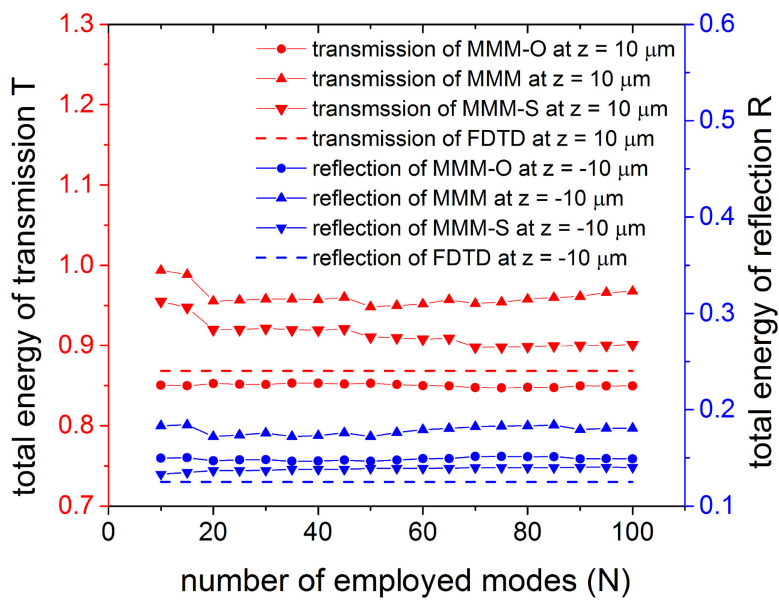

Fig. 13. The total energy of transmission (reflection) at $z=10 \mu \mathrm{m}(z=$ $-10 \mu \mathrm{m})$ of MMM, MMM-S, MMM-O and FDTD.

based on [21]. The sizes and refractive indexes of the fiber and SOI are shown in Figs. 11 and 12.

The comparison of the total energy of transmission (reflection) at $z=10 \mu \mathrm{m}(z=-10 \mu \mathrm{m})$ calculated by MMM-O, MMM, MMM-S and FDTD are shown in Fig. 13. If we regard the results of FDTD as a benchmark, the figure demonstrates that MMM-O has the minimum error in transmission, while MMM-S has the minimum error in reflection. Considering the accuracy of transmission and reflection at the same time, MMM$\mathrm{O}$ is undoubtedly the best choice. It makes both errors relatively small.

\section{DisCUSSION AND PMLS IN Mode ANALYSIS}

The PML technique is widely used to truncate the infinite domain in numerical simulations. It can be applied in the modal expansion of open waveguides, which are unbounded in the transverse plane. There are several numerical methods to calculate the modes of the bounded waveguide with PML, such as the finite difference method, finite element method and the rigorous coupled wave analysis (RCWA). In [22]-[24] the completeness of the modes for plate waveguides, fibers and three-dimensional structures with PMLs was proven, respectively. Theoretically, PMLs should absorb all incident waves without reflection, but the PMLs still have some reflection after being discretized [25].

The determination of PML parameters affects the numerical accuracy of the modes of open waveguides and, as a consequence, it affects the transmission and reflection coefficients based on these modes. The modes with discrete spectra obtained by numerical calculation can by roughly divided into two categories, i.e., guided modes and complex modes. Complex modes can be seen as the packets of radiation modes [23] and they are not orthogonal in the real domain [15], especially those modes that exist mainly in the PMLs. As the number of this type of mode increases, the condition number of the coefficient matrix increases and the singularity of the same matrix is intensified, resulting in the deviations in the figures obtained from MMM. Naturally, we look for ways to get an optimal solution without the interference caused by the singular nature of the coefficient matrix. Fortunately, with the conservation of energy as a constraint, we successfully avoid this singularity and achieved good results.

\section{CONCLUSION}

We proposed an improved method to solve the equations obtained from MMM. By introducing the constraint condition based on the conservation of energy and adding it to the object function as a penalty, the equations are solved as an unconstrained optimization problem. Although compared with MMM and MMM-S, the computational cost of our proposed method is several times higher than that of MMM / MMM-S, our method yields more stable convergence and smaller field-mismatch error. It is suitable for a larger range of mode selection and geometric discontinuity.

\section{REFERENCES}

[1] D. F. Gallagher and T. P. Felici, "Eigenmode expansion methods for simulation of optical propagation in photonics: Pros and cons," in Proc. Integ. Optoelectronics Devices, 2003, pp. 69-82.

[2] G. V. Eleftheriades, A. S. Omar, L. P. Katehi, and G. M. Rebeiz, "Some important properties of waveguide junction generalized scattering matrices in the context of the mode matching technique," IEEE Trans. Microw. Theory Techn., vol. 42, no. 10, pp. 1896-1903, Oct. 1994.

[3] M. Reed, P. Sewell, T. Benson, and P. Kendall, "Efficient propagation algorithm for 3D optical waveguides," Inst. Electron. Eng. Proc.Optoelectronics, vol. 145, no. 1, pp. 53-58, Feb. 1998.

[4] K. Jiang, "Analysis of waveguide discontinuities by mode matching method," Ph.D. dissertation, Dept. Elect. Eng., McMaster Univ., Hamilton, ON, Canada, 2006.

[5] E. Anemogiannis and E. N. Glytsis, "Multilayer waveguides: Efficient numerical analysis of general structures," J. Lightw. Technol., vol. 10, no. 10, pp. 1344-1351, Oct. 1992. 
[6] L. Li, "Multilayer modal method for diffraction gratings of arbitrary profile, depth, and permittivity," J. Opt. Soc. Amer. A, vol. 10, no. 12, pp. 25812591, 1993.

[7] Y. Jiao, Y. Shi, D. Dai, and S. He, "Accurate and efficient simulation for silicon-nanowire-based multimode interference couplers with a 3D finiteelement mode-propagation analysis," J. Opt. Soc. Amer. B, vol. 27, no. 9, pp. 1813-1818, 2010 .

[8] K. P. Fakhri and P. Benech, "A new technique for the analysis of planar optical discontinuities: An iterative modal method," Opt. Commun., vol. 177 , no. 1 , pp. 233-243, 2000.

[9] H. Liang, J. Mu, R. A. Soref, X. Li, and W.-P. Huang, "An optical modematching method with improved accuracy and efficiency," IEEE J. Quant. Electron., vol. 51, no. 2, pp. 1-8, Feb. 2015.

[10] A. W. Snyder and J. Love, Optical Waveguide Theory. Berlin, Germany: Springer Science \& Business Media, 2012.

[11] J. Mu and W.-P. Huang, "Simulation of three-dimensional waveguide discontinuities by a full-vector mode-matching method based on finitedifference schemes," Opt. Exp., vol. 16, no. 22, pp. 18 152-18 163, 2008.

[12] P. Bienstman and R. Baets, "Optical modelling of photonic crystals and VCSELs using eigenmode expansion and perfectly matched layers," Opt. Quantum Electron., vol. 33, no. 4/5, pp. 327-341, 2001.

[13] K. Jiang and W.-P. Huang, "Finite-difference-based mode-matching method for 3-D waveguide structures under semivectorial approximation," J. Lightw. Technol., vol. 23, no. 12, pp. 4239-4248, Dec. 2005.

[14] S. D. Gedney and B. Zhao, "An auxiliary differential equation formulation for the complex-frequency shifted PML," IEEE Trans. Antennas Propag., vol. 58, no. 3, pp. 838-847, Mar. 2010.

[15] W.-P. Huang and J. Mu, "Complex coupled-mode theory for optical waveguides," Opt. Express, vol. 17, no. 21, pp. 19 134-19 152, 2009.

[16] W. Sun and Y.-X. Yuan, Optimization Theory and Methods: Nonlinear Programming, vol. 1. Berlin, Germany: Springer Science \& Business Media, 2006.

[17] K. Saitoh and M. Koshiba, "Full-vectorial finite element beam propagation method with perfectly matched layers for anisotropic optical waveguides," J. Lightw. Technol., vol. 19, no. 3, pp. 405-413, Mar. 2001.

[18] J.-M. Jin, The Finite Element Method in Electromagnetics. Hoboken, NJ, USA: Wiley, 2015

[19] R. A. Horn and C. R. Johnson, Matrix Analysis. Cambridge, U.K.: Cambridge Univ. Press, 2012.

[20] Solution, F. D. T. D. Professional commercial software from Lumerical Solution Inc., Canada. [Online]. Available: http://www.lumerical.com

[21] A. Barkai et al., "Double-stage taper for coupling between SOI waveguides and single-mode fiber," J. Lightw. Technol., vol. 26, no. 24, pp. 38603865, Dec. 2008

[22] L. F. Knockaert and D. De Zutter, "On the completeness of eigenmodes in a parallel plate waveguide with a perfectly matched layer termination," IEEE Trans. Antennas Propag., vol. 50, no. 11, pp. 1650-1653, Nov. 2002.

[23] B. Huang, L. Yang, Y.-L. Tian, and J.-R. Qian, "Intuitive equivalence between radiation modes and quasi-leaky modes in optical waveguides," J. Lightw. Technol., vol. 35, no. 9, pp. 1640-1645, May 2017.

[24] F. Olyslager, "Discretization of continuous spectra based on perfectly matched layers," SIAM J. Appl. Math., vol. 64, no. 4, pp. 1408-1433, 2004

[25] D. C. Wittwer and R. W. Ziolkowski, "How to design the imperfect berenger PML," Electromagnetics, vol. 16, no. 4, pp. 465-485, 1996.
Zizheng Cao received the B.Sc. degree in electronic information science and technology from Hunan Normal University, Changsha, China, the Master of Engineering in telecom engineering (awarded Outstanding thesis of master degree of Hunan Province) from Hunan University, Changsha, China, in 2010, and the Ph.D. degree with highest honors from the Eindhoven University of Technology (TU/e), Eindhoven, The Netherlands, in 2015. He is an Assistant Professor with the Department of Electrical Engineering, TU/e. His current research interest include modeling and design of integrated photonics circuits, microwave photonics, advanced DSP, and physical layer design of optical network. Practica application examples include cascaded aperture optical receivers which overcome capacitance limitations inherent to traditional photodiode designs and can provide the basis for an indoor optical wireless communication system.

M. C. van Beurden studied electrical engineering with the Eindhoven University of Technology, Eindhoven, The Netherlands, where he received the M.Sc degree (cum laude) in 1997. In 2003, he received the Ph.D. degree (cum laude) He then started the Ph.D. research with the Eindhoven University of Technology (TU/e), in ), under the supervision of Professors A. Tijhuis and P. Zwamborn In 2000, he conducted research on the optimization of wire antennas with the Department of Mathematics, University of Dundee with Professors P. Smith and R. Fletcher. In September 2000, he was appointed an Assistant Professor, TU/e. Since 2012, he has been an Associate Professor with the Department of Electrical Engineering, Electromagnetics group of the TU/e. His research interests include fast modeling techniques, integral equations, functional analysis with respect to electromagnetic field problems, inverse scattering, and nonlinear optimization. His current projects involve, amongst others, forward and inverse scattering for periodic structures and finitely repetitive structures in layered media; modeling of electromagnetic fields in configurations with a stochastic or uncertain nature; and the design and optimization of antennas. He was the recipient of the ASML prize for the best Ph.D. thesis with applied research, TU/e, in 2004

Yuqing Jiao received dual Ph.D. degrees both from the Eindhoven University of Technology (TU/e), Eindhoven, The Netherlands and Zhejiang University, Hangzhou, China, in 2013. He has continued his research with the TU/e, where he has been working as an Assistant Professor since 2016. He is an Assistant Professor with the Institute for Photonic Integration (IPI, formerly COBRA Research Institute), Eindhoven University of Technology, TU/e. His key fields of expertise include photonic integrated circuits (PICs), their manufacturing technologies and application exploitations. He is currently leading the research activities on advanced nanophotonic PICs. He is focusing on key enabling technologies for manufacturable nanophotonics.

Qingbiao Wu works as a Professor, Deputy Director of the Institute of Science and Engineering Computing and Assistant Director of the Institute of Industrial Technology with Zhejiang University. His main research interests include nonlinear numerical algebra, nonlinear numerical optimization, large data analysis, computer graphics and image processing, numerical calculation method, Monte Carlo method, and computer simulation.

Ton Koonen received the M.Sc. (with honors) degree in electrical engineering from the Eindhoven University of Technology, Eindhoven, The Netherlands, in 1979. In that year, he was Philips Telecommunicatie Industrie (Telecommunications Industry). From 1987 to 2000, he was on high-speed transmission systems and optical fiber systems for hybrid access networks, Bell Laboratories within Lucent. He was also a Professor with the University of Twente, holding a chair on Photonic Networks. He is a Chairman of the Electro-Optical Communication Systems group, part of the COBRA Institute, and from September 2012, he was also vice-dean of the Department Electrical Engineering. His areas of specialization include computer systems, architectures and networks, telecommunications, broadband, and optical fiber-to/in-the-home. His current research interests include optical fiber-supported in-building networks (including optical wireless communication techniques, radio-over-fiber techniques and high-capacity plastic optical fiber techniques), optical access networks, and spatial division multiplexed systems.
Xinyang Lu received the B.S. degree in mathematics from Zhejiang University, Hangzhou, China, in 2014. She is currently working toward the Ph.D. degree in mathematics from Zhejiang University. In 2016 to 2018, she joined the Electro-Optical Communication Systems Group with the Eindhoven University of Technology as a visiting Ph.D. student. Her research interest includes the numerical simulation of light propagation in 3-D waveguides and solver of electromagnetic scattering problems in dielectric layered media. 